\title{
A Comparison of Static Stretching Versus Combined Static and Ballistic Stretching in Active Knee Range of Motion
}

\author{
Nathan R. Blackhurst \\ Yuma Union High School District \#70, nateblackhurst@gmail.com \\ James C. Peterson \\ Integrity Rehab Group, Inc, christianpeterson1@mail.weber.edu \\ Valerie W. Herzog \\ Weber State University, ValerieHerzog@weber.edu \\ Ericka P. Zimmerman \\ University of Charleston, epzimmerman@wcu.edu
}

Follow this and additional works at: https://nsuworks.nova.edu/ijahsp

Part of the Other Rehabilitation and Therapy Commons, Sports Medicine Commons, and the Sports Sciences Commons

\section{Recommended Citation}

Blackhurst NR, Peterson JC, Herzog VW, Zimmerman EP. A Comparison of Static Stretching Versus Combined Static and Ballistic Stretching in Active Knee Range of Motion. The Internet Journal of Allied Health Sciences and Practice. 2015 Oct 20;13(4), Article 11.

This Manuscript is brought to you for free and open access by the College of Health Care Sciences at NSUWorks. It has been accepted for inclusion in Internet Journal of Allied Health Sciences and Practice by an authorized editor of NSUWorks. For more information, please contact nsuworks@nova.edu. 


\title{
A Comparison of Static Stretching Versus Combined Static and Ballistic Stretching in Active Knee Range of Motion
}

\begin{abstract}
Background: There is continued controversy related to flexibility gains from different stretching protocols and within single protocols. Stretching methods include static, ballistic, dynamic, and proprioceptive neuromuscular facilitation (PNF). A combination of stretching methods may be an improved way to increase active knee range of motion (ROM). This study evaluated a single program formulated with static and ballistic components. Objective: To compare active knee ROM following stretching programs which either included combined static and ballistic stretching (CSBS) or static stretching (SS) alone. It was hypothesized that CSBS would show a greater increase in active knee ROM than SS. Setting: The pre- and post- measurements were performed in a laboratory. Subjects were randomly assigned to either treatment group or a non-stretching control group and given written instructions on how to perform their designated protocol at home. Subjects: Forty-three $(33 \mathrm{M}, 10 \mathrm{~F})$ healthy collegiate aged participants $(24.0+3.69 \mathrm{yrs}$, $176.21+10.0 \mathrm{~cm}, 78.15+12.93 \mathrm{~kg}$ ) with no history of injury to the lower extremity or low back for the previous 6 months were eligible to participate in the study. Interventions: Two treatment groups either performed SS or CSBS for 30 seconds on each leg, twice a day for 2 weeks. All subjects but 3 provided both legs, and each leg was evaluated separately, providing 83 total measurements. Main Outcome Measures: A Johnson Digital Inclinometer was used to measure active knee extension. A mixed ANOVA with a Tukey post hoc test was used for statistical analysis. Results: There was no statistically significant difference in active knee ROM between groups at the pre-test, $F(2,80)=1.062, p=.351$, partial $\eta 2=.026$ (SS: $52.56+7.50^{\circ}$, CSBS: $49.84+8.91^{\circ}$, control: $\left.49.39+10.09^{\circ}\right)$. There was a statistically significant difference in active knee ROM between groups at the post-test, $F(2,80)=29.034, p .05)$. There was homogeneity of covariance's, as assessed by Box's test of equality of covariance matrices $(p=.076)$. There was homogeneity of variances, as assessed by Levene's test of homogeneity of variance ( $p>05$ ). Conclusions: SS and CSBS are equally effective for improving active knee ROM. A trend indicating CSBS showing only slightly greater differences may be due to limited time allowed to master the CSBS method, with no supervision during stretching sessions.
\end{abstract}

\section{Author Bio(s)}

- Nathan Blackhurst, MS, LAT, ATC, is the Head Athletic Trainer at San Luis High School, a $5 A$ school in the Yuma, AZ area. His full-time employment includes teaching the Career and Technical Education Sports Medicine and Rehabilitation Services program.

- Valerie W. Herzog, EdD, LAT, ATC, is an Associate Professor of Athletic Training and also serves as the Graduate Athletic Training Program Director and the Director of the Office of Graduate Studies at Weber State University in Ogden, UT. Her research focuses on static balance and proprioception.

- James C. Peterson, BS, is a current medical student at Oakland University William Beaumont School of Medicine. He is an Ambassador of the school's Orthopedic Surgery and Sports Medicine Group and is pursuing research in the field of Orthopedics. Prior to his medical education, he received his Bachelor of Science at Weber State University, with a focus on Athletic Therapy.

- Ericka P. Zimmerman, EdD, LAT, ATC, CES, PES, is an Associate Professor and Director for the School of Health Sciences at Western Carolina University in Cullowhee, NC. 


\title{
TIAHSP \\ The Internet Journal of Allied Health Sciences and Practice \\ Dedicated to allied health professional practice and education
}

Vol. 13 No. 4 ISSN 1540-580X

\section{A Comparison of Static Stretching Versus Combined Static and Ballistic Stretching in Active Knee Range of Motion}

\author{
Nathan R. Blackhurst, MS, LAT, ATC1 \\ J. Christian Peterson, BS 2 \\ Valerie Herzog, EdD, LAT, ATC ${ }^{3}$ \\ Ericka P. Zimmerman, EdD, LAT, ATC, CES, PES ${ }^{4}$
}

1. Yuma Union High School District \#70, Yuma, Arizona

2. Oakland University School of Medicine student, Rochester, Michigan

3. Graduate Athletic Training Program Director, Department of Athletic Training and Nutrition, Weber State University, Ogden, UT

4. Associate Professor \& Director, School of Health Sciences, Western Carolina University, Cullowhee, NC

United States

\begin{abstract}
Background: There is continued controversy related to flexibility gains from different stretching protocols and within single protocols. Stretching methods include static, ballistic, dynamic, and proprioceptive neuromuscular facilitation (PNF). A combination of stretching methods may be an improved way to increase active knee range of motion (ROM). This study evaluated a single program formulated with static and ballistic components. Objective: To compare active knee ROM following stretching programs which either included combined static and ballistic stretching (CSBS) or static stretching (SS) alone. It was hypothesized that CSBS would show a greater increase in active knee ROM than SS. Setting: The pre- and postmeasurements were performed in a laboratory. Subjects were randomly assigned to either treatment group or a non-stretching control group and given written instructions on how to perform their designated protocol at home. Subjects: Forty-three (33M, $10 \mathrm{~F})$ healthy collegiate aged participants $(24.0 \pm 3.69 \mathrm{yrs}, 176.21 \pm 10.0 \mathrm{~cm}, 78.15 \pm 12.93 \mathrm{~kg})$ with no history of injury to the lower extremity or low back for the previous 6 months were eligible to participate in the study. Interventions: Two treatment groups either performed SS or CSBS for 30 seconds on each leg, twice a day for 2 weeks. All subjects but 3 provided both legs, and each leg was evaluated separately, providing 83 total measurements. Main Outcome Measures: A Johnson Digital Inclinometer was used to measure active knee extension. A mixed ANOVA with a Tukey post hoc test was used for statistical analysis. Results: There was no statistically significant difference in active knee ROM between groups at the pre-test, $F(2,80)=1.062, p=.351$, partial $\eta 2=.026$ (SS: $52.56 \pm 7.50^{\circ}$, CSBS: $49.84 \pm 8.91^{\circ}$, control: $49.39 \pm 10.09^{\circ}$ ). There was a statistically significant difference in active knee ROM between groups at the post-test, $F(2,80)=29.034, p<.001$, partial $\eta 2=.421$, with SS $\left(67.21 \pm 8.23^{\circ}\right)$ and CSBS $\left(65.32 \pm 7.89^{\circ}\right)$ greater than the control group $\left(49.30 \pm 12.33^{\circ}\right)$. However, the two stretching programs were not statistically significantly different from one another $(p=.767,>.05)$. There was homogeneity of covariance's, as assessed by Box's test of equality of covariance matrices $(p=.076)$. There was homogeneity of variances, as assessed by Levene's test of homogeneity of variance ( $p>.05)$. Conclusions: SS and CSBS are equally effective for improving active knee ROM. A trend indicating CSBS showing only slightly greater differences may be due to limited time allowed to master the CSBS method, with no supervision during stretching sessions.
\end{abstract}

\section{INTRODUCTION}

The ability of an individual to move a given joint through a functional active range of motion (ROM) is one of many critical components for fitness, sports, and activities of daily living. Certain athletes often require increased motion beyond normal ranges in order to optimize performance, but since most athletic activities require movements within normal ranges, excessive flexibility is not necessary to perform required skills or to reduce the risk of injury. ${ }^{1}$ For athletes requiring motion beyond normal 
ranges, or for athletes who suffer from limited ROM, compliancy of associated muscle or muscle groups may bring about these desired increases. ${ }^{1}$ As the muscle-tendon unit becomes more compliant, the risk of injury may decrease as a result of increased absorption of energy which then delays and reduces force production. ${ }^{1,2}$ Wong et al concluded that by incorporating dynamic stretching into a static stretching program, compliancy can be achieved without compromising force production, thereby minimizing the risk for injury, while range of motion and force production are maximized. ${ }^{3}$ Contrary to this, Little and Williams concluded static stretching by itself is not detrimental to high-speed performance when included in a warm-up. ${ }^{4}$ As with most research on different modes of stretching, there is controversy about whether a stretching program will in fact reduce the incidence of injuries. ${ }^{5}$

There are many stretching methods used that have demonstrated increased flexibility. Among the better known methods are: static, ballistic, dynamic, and proprioceptive neuromuscular facilitation (PNF). Due to inconsistencies in the parameters used in the published research, such as duration, frequency, intensity, and length of program, it is difficult to conclude whether one method is superior to another. Static stretching is arguably the most widely used method, possibly due to its simplistic technique and low risk of injury. Bandy et al studied the differences between a 30- and 60-second static stretch, performed one and three times daily for 6 weeks. ${ }^{6}$ All treatments showed improvements against the control; however, they found no significant difference to the ROM of the hamstring muscle in terms of duration and frequency between treatment groups. ${ }^{6}$ Bandy et al concluded that one 30 -second stretch is as efficacious as three 60-second stretches with ten-second rest periods in between. Ogura et al also studied 30-second and 60-second static stretches, similarly finding significant results for both compared to a control, yet no difference between the two treatment groups. ${ }^{2}$ Ogura et al also evaluated potential force production changes within these groups. ${ }^{2}$ The 30-second group suffered no force production losses, whereas the 60 -second group found force production to be compromised. ${ }^{2}$ Bandy et al and Ogura et al have provided support for static stretching of 30-seconds once daily. 2,6 Lasting effects of stretching can identify a guide for the frequency of a stretching program. de Weijer et al studied the lasting effect of a static stretch on hamstring flexibility and concluded flexibility can be maintained for up to 24 hours, a finding also identified in a systematic review by Harvey et al. ${ }^{7,8}$ This research supports a once daily stretching routine.

Ballistic stretching traditionally is used less often than static stretching, except when considering the similarities between this method and dynamic stretching. Dynamic stretching, which is widely used by collegiate athletes, is performed by repeating forceful sport-specific movements, whereas ballistic stretching is thought of as repeated bouncing maneuvers, not necessarily as a sport-specific motion. ${ }^{9}$ However, both methods contain ballistic traits. Ballistic stretching has been negatively labeled by fitness professionals as potentially harmful when not performed correctly, most likely due to the incidence of injuries received by the less-trained general public. Despite this perception, Kumar and Chakrabarty have shown ballistic stretching to be more effective than static stretching, when performed properly, for increasing ROM. ${ }^{10}$

The conflicting evidence as to which stretching method is superior warrants further investigation. Because no single program has shown to be clearly superior, perhaps combining two stretching techniques, static and ballistic, can show superiority over a single technique; in this case, the program with the widest use-static stretching. This combination takes advantage of the statically induced golgi tendon organ (GTO) activation, which is a muscle inhibitory response to static tension, either by active contraction or by passive stretching. ${ }^{11-14}$ This inverse myotatic reflex is caused by the muscle collagen braids applying direct pressure to the type $1 \mathrm{~b}$ afferent nerve fibers that innervate the GTOs, causing relaxation to the antagonistic muscle being stretched.11-14 Immediately following GTO activation, performing repeated gentle ballistic bouts is theorized to mildly increase the ROM endpoint felt with static stretching without being held long enough to damage the muscle. As a result, the ballistic motions may allow a temporary compliancy for a second static stretch to be held at the further endpoint, without engaging the antagonistic musculature in a protective myotatic reflex, increasing ROM beyond what a static stretch alone could allow. Therefore, the purpose of this study was to determine if a combined static and ballistic stretching protocol produces significant gains compared to static stretching alone. In this study, participants performed 30-second stretches, twice a day for 2 weeks

\section{METHODS}

The efficacy of a 2-week combined static-ballistic stretching program when compared to static stretching on active knee ROM was examined in this study. Subjects recruited had both right and left lower limbs examined in order to increase the number of measurements. Participants were randomly assigned to 1 of 3 groups: static stretching (SS), combined static and ballistic stretching (CSBS), and a non-stretching control. Once assigned, those in SS and CSBS groups were provided specific written protocol instructions and a youtube.com link visually demonstrating the assigned protocol. This was to ensure consistency of quality of the instructions given to both groups, as well as to provide assistance in continued proper execution since the stretching sessions were performed twice-daily for 2 weeks without direct monitoring. Reminder text messages were sent to each participant every morning and evening during the course of the study to reduce the incidence of missed sessions and to maximize subject retention. Daily logs were provided for subjects to mark off completion of each of the 28 total stretching

(c) The Internet Journal of Allied Health Sciences and Practice, 2015 
sessions throughout the treatment period. These logs were returned at the time of post-testing, signed, documenting full adherence to the study. Any participants who missed more than three stretching sessions were excluded from the study. Effectiveness was determined by measuring active knee extension ROM on the day of, and prior to, the first session (pre-test) and the day of, and following, the last session (post-test). In an attempt to control for active knee ROM differences that can present at differing times of day, each subject's post-test was scheduled for the same time of day as their initial pre-test appointment time.

\section{Subjects}

Following Institutional Review Board approval, a convenience sample of 43 male and female participants provided written informed consent. The 43 subjects (33 male, 10 female, $24.0 \pm 3.69 \mathrm{yrs}, 176.21 \pm 10.0 \mathrm{~cm}, 78.15 \pm 12.93 \mathrm{~kg}$ ) completed the study and were randomly assigned to one of three groups (SS, $n=16$; CSBS, $n=12$; control, $n=15$ ) providing a total of 83 measurements, including 3 subjects with only unilateral qualification as a result of one limb showing active knee ROM to be lacking less than $20^{\circ}$ knee extension. Inclusion criteria required all participants to have at least a $20^{\circ}$ lack of full active knee extension when the hip is flexed to $90^{\circ}$, be between ages 18 to 35 , and have self-reported normal knee and hip function. Exclusion criteria included having had any low back or lower limb neuromusculoskeletal injuries in the preceding 6 months, stretching that is additional beyond the study requirements, including exercises that promote ROM of the hips or knees (i.e. deep squat), pregnancy, and any known chronic disease or illness. Subjects were asked to refrain from beginning a physical activity program or increase current levels of physical activity. Among the four subjects who did not complete the study, 3 failed to arrive for their scheduled post-measurement appointment, and 1 was disqualified for increasing physical activity levels and performing stretches beyond the study requirements.

\section{Measurement Procedures}

For pre- and post-testing, all subjects arrived wearing athletic shorts, allowing for accessibility to the anterior thigh and lower leg, and to reduce erroneous resistance to active knee extension. Subjects were instructed to wear the same clothing for the pre and posttest measurements for consistency. One researcher (Researcher 1) positioned the subject supine on a plinth. A lower limb stabilizing device made of polyvinylchloride piping and wood was placed over the hips. The subject was then instructed to flex both hips with the knees positioned at approximately $90^{\circ}$ until contact was made with the anterior aspect of the distal thigh against the device. The researcher then placed a right-angled box made of particle board under the heels of the subject to help maintain bilateral $90^{\circ}$ hip flexion and to reduce lumbar lordosis and unilateral pelvic tilt. A second researcher (Researcher 2) then measured hip flexion of both limbs using a Johnson Digital Inclinometer, with a reported accuracy of $+/-0.1$ degrees within 0 to 90 degrees, placed on the anterior thighs, directly over the rectus femoris muscle. Once a reading of $90^{\circ}$ hip flexion was taken, Researcher 2 maintained the position of the limb while Researcher 1 adjusted the lower limb stabilizing device to set the hip in this position during the measuring process. Researcher 2 was then able to release the limb since the device was in place, and then proceeded to set the digital goniometer adjacent to the distal surface of the tibial tuberosity, resting it upon the longitudinal surface of the tibial crest. During this time, Researcher 1 instructed the subject to wait for the cue from Researcher 2, at which point proceeded to slowly extend the knee of the limb being measured actively, to the hamstring muscles' endpoint, but not to the point of pain, and to hold that position until Researcher 2 captured the measurement (Figure 1). Each measurement was taken twice and the average of the two measurements were used in the analysis. Once the subject returned to the original bilateral $90 / 90$ position, this procedure was then repeated for the opposite limb, starting with measuring the hip at $90^{\circ}$ and readjusting the lower limb stabilizing device.

\section{Figure 1. Measurement Procedure Displaying Subject Positioning, Lower Limb Stabilizing Device, and Right-Angled Box}

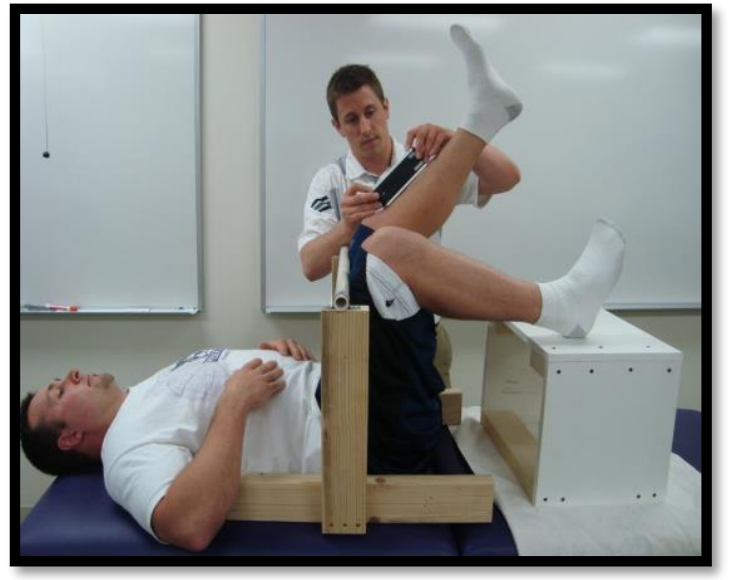

(C) The Internet Journal of Allied Health Sciences and Practice, 2015 


\section{Stretching Procedures}

For the purpose of this study, and to help translate the findings to be used by an athletic population, 30-second treatments were given to SS and CSBS groups. A modified hurdler position was performed for the designated length of time each morning and evening for 2 weeks (Figure 2a and 2b).

\section{Static.}

Subjects performed a thirty-second operation. Each subject was asked to sit in a modified hurdler position described as having the stretching leg fully extended with the ankle relaxed, the non-stretching leg flexed with the sole of the foot against the medial side of the knee of the stretching leg, and the toes lined up to the joint line. The hands were then placed directly on each side of the stretching leg, positioned with the thumb side up and the pinky side making contact with the ground, with elbows fully extended. The subject then flexed the hips and lumbar spine, while maintaining as neutral as possible (not flexed) thoracic and cervical spine, allowing the hands to slide down the sides of the leg towards the foot, to a point which remains uncomfortable yet tolerable, and would become painful by flexing any further. This single, static position was held for 30-seconds to allow for comparable times in a maximally stretched position for SS and CSBS groups.

\section{Combined Static and Ballistic.}

Subjects performed a 30-second operation, divided into 3 consecutive 10-second phases; static, ballistic, and static.

Phase 1. Each subject was asked to perform the static stretch as described in the static stretching procedures. This static position was held for 10-seconds.

Phase 2: Five repeated gentle ballistic movements were executed. The ballistic movements were performed continuously in a gentle and controlled manner, with the muscle lengthening and shortening portions of the stretch each lasting 1 second (s), totaling at 2 seconds of continuous motion to complete 1 full ballistic movement beginning and ending in the stretched position found during phase 1 . Beginning exactly at the 10 second mark, the first ballistic motion was executed by flexing the trunk forward beyond the point maintained during phase 1, as far as can be tolerated without pain for a 1-second period, but would likely cause pain if it was held for several seconds. Therefore, each attempt to lengthen the muscle occurred at $10 \mathrm{~s}, 12 \mathrm{~s}, 14 \mathrm{~s}$, $16 \mathrm{~s}$, and 18s, while each attempt to return the muscle tension back to the original phase 1 endpoint occurred at $11 \mathrm{~s}, 13 \mathrm{~s}, 15 \mathrm{~s}$, $17 \mathrm{~s}$, and 19s. Upper body positioning remained constant, allowing for the hands to slide back and forth during gentle ballistic trunk flexion.

Phase 3. A second static stretch for the remaining 10 seconds was then performed at the 20 second mark. This final position was held at the furthest point achieved during the ballistic movements found in phase 2. At the 30-second mark, each subject then returned to a fully relaxed, upright position.

\section{Figure 2a. Starting Position for SS and CSBS Protocols}

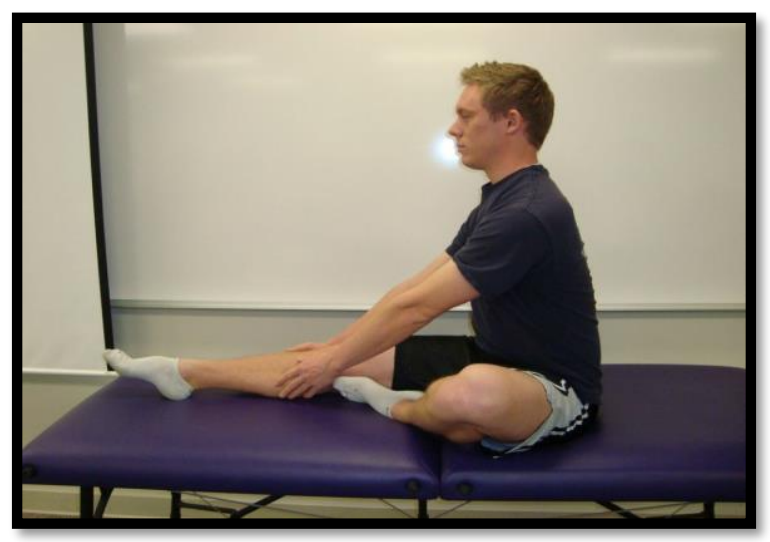


Figure 2b. Ending Position for SS Protocol; Phase 1 Position for CSBS Protocol

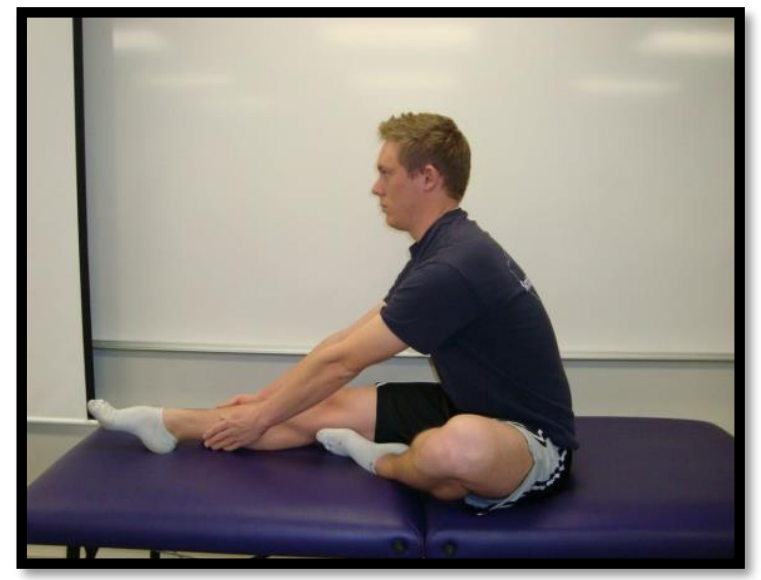

Control. The control group was non-stretching, but was to adhere to the same inclusion criteria, including that of physical activity levels during their 2-week participation.

\section{Statistical Analysis}

Statistical analysis was performed using Windows software, Statistical Package for the Social Services (SPSS) version 20. A mixed factorial $2 \times 3$ analysis of variance was used to determine the interaction effect with the independent variables including a pre-post repeated measure factor and a between-groups factor. A post hoc Tukey test was used to test for pairwise differences, and all significance levels were set at 0.05 .

\section{RESULTS}

Data analysis revealed that there was no statistically significant difference in active knee ROM between groups at the pre-ROM measurement, $F(2,80)=1.062, p=.351$, partial $\eta 2=.026$. Active knee ROM gains were statistically significantly greater in the SS group $\left(M=17.90^{\circ}, S E=2.56^{\circ}, p<.001\right)$ and the CSBS group $\left(M=16.02^{\circ}, S E=2.69^{\circ}, p<.001\right)$ compared to the control group. However, the active knee ROM gains were not statistically significantly different between the two different stretching groups $\left(\mathrm{M}=1.88^{\circ}, \mathrm{SE}=2.71^{\circ}, \mathrm{p}=.767\right.$ ) (see Table 1). The main effect for time showed a statistically significant difference in active knee ROM at the different time points (pre vs. post), $F(1,80)=140.991$, $p<.001$, partial $\eta 2=.638$. There was a statistically significant interaction between the stretching programs and time on active knee $R O M, F(2,80)=37.526, p<.001$, partial $\eta 2=.484$. The main effect for groups showed there was a statistically significant difference in active knee ROM between each group $F(2,80)=12.220$, $p<.001$, partial $\eta 2=.234$. There was a statistically significant difference in active knee ROM between groups at the post-ROM measurement, $F(2,80)=29.034, p<.001$, partial $\eta 2=.421$.

There was a statistically significant effect of time on active knee ROM for the SS group, $F(1,28)=132.983$, $p<.001$, partial $\eta 2=.826$. Participants had a statistically significant increase in their ROM from the pre to the post measurement $\left(M=14.647^{0}\right.$, $\mathrm{SE}=1.270^{\circ}, p<.001$ ). There was a statistically significant effect of time on active knee ROM for the CSBS group, $F(1,23)=69.308$, $p<.001$, partial $\eta 2=.751$. Participants had a statistically significant increase in their active knee ROM from the pre to the post measurement $\left(M=15.481^{\circ}, S E=1.860^{\circ}, p<.001\right)$. There was no statistically significant effect of time on active knee ROM for the control group, $F(1,29)=.005, p=.944$, partial $\eta 2<.001$. Participants had no statistically significant change in their active knee ROM from the pre to the post measurement $\left(\mathrm{M}=.092^{\circ}, \mathrm{SE}=1.294^{\circ}, \mathrm{p}=.944\right)$. There was homogeneity of variances, as assessed by Levene's test of homogeneity of variance $(p>.05)$. There was homogeneity of covariance's, as assessed by Box's test of equality of covariance matrices $(p=.076)$.

Table 1. Pre- and post-test measurements for active ROM of the knee.

\begin{tabular}{|l|l|l|l|}
\hline & $\mathrm{N}$ & Pre & Post \\
\hline Static Stretching (SS) & 29 & $52.56 \pm 7.50$ & $67.21 \pm 8.23$ \\
\hline $\begin{array}{l}\text { Combined Static \& Ballistic } \\
\text { Stretching (CSBS) }\end{array}$ & 24 & $49.84 \pm 8.91$ & $65.32 \pm 7.89$ \\
\hline Control & 30 & $49.39 \pm 10.09$ & $49.30 \pm 12.33$ \\
\hline
\end{tabular}

(c) The Internet Journal of Allied Health Sciences and Practice, 2015 


\section{DISCUSSION}

Combining static and ballistic elements into one structured method of stretching, in order to improve active knee ROM, is equal in effectiveness to static stretching alone (Figure 3). A trend showing that CSBS may be more effective than static stretching was noted. Perhaps by using a larger subject population to increase statistical power, with equal numbers of participants in each group, including only those who are skilled and experienced with stretching, allowing a period of time to master a CSBS protocol prior to initiating the program, and/or if a treatment period greater than two weeks was implemented, results may have shown that CSBS is statistically significantly more effective than static stretching. This is possible since those who are more skilled at stretching better understand how subjective ROM endpoints feel, and may be more likely to flex the trunk further during a bout if they are more experienced with feelings of discomfort while stretching, which during the course of a program may contribute to greater results over time.

The results of this investigation provided further evidence supporting the efficacy of stretching in order to increase active knee ROM. Through clinical research, static stretching has repeatedly been demonstrated to increase passive ROM, however, to inconsistent degrees. $6,15,16$ This is based on differing parameters, such as duration, frequency, intensity, and length of program. Bandy et al determined the optimal duration for a single static stretching bout to be 30 seconds. ${ }^{6}$ Our research supports these findings by showing significant gains with 30 -second stretches in both intervention groups. This is despite evaluating a stretching method not exclusively performed statically, as well as both methods used in this study being executed twice daily, every day for 2 weeks, rather than once daily, 5 days a week for 6 weeks, as shown by Bandy et al. ${ }^{6}$

\section{Figure 3. Combined Static and Ballistic Stretching is Equally Effective as Static Stretching}

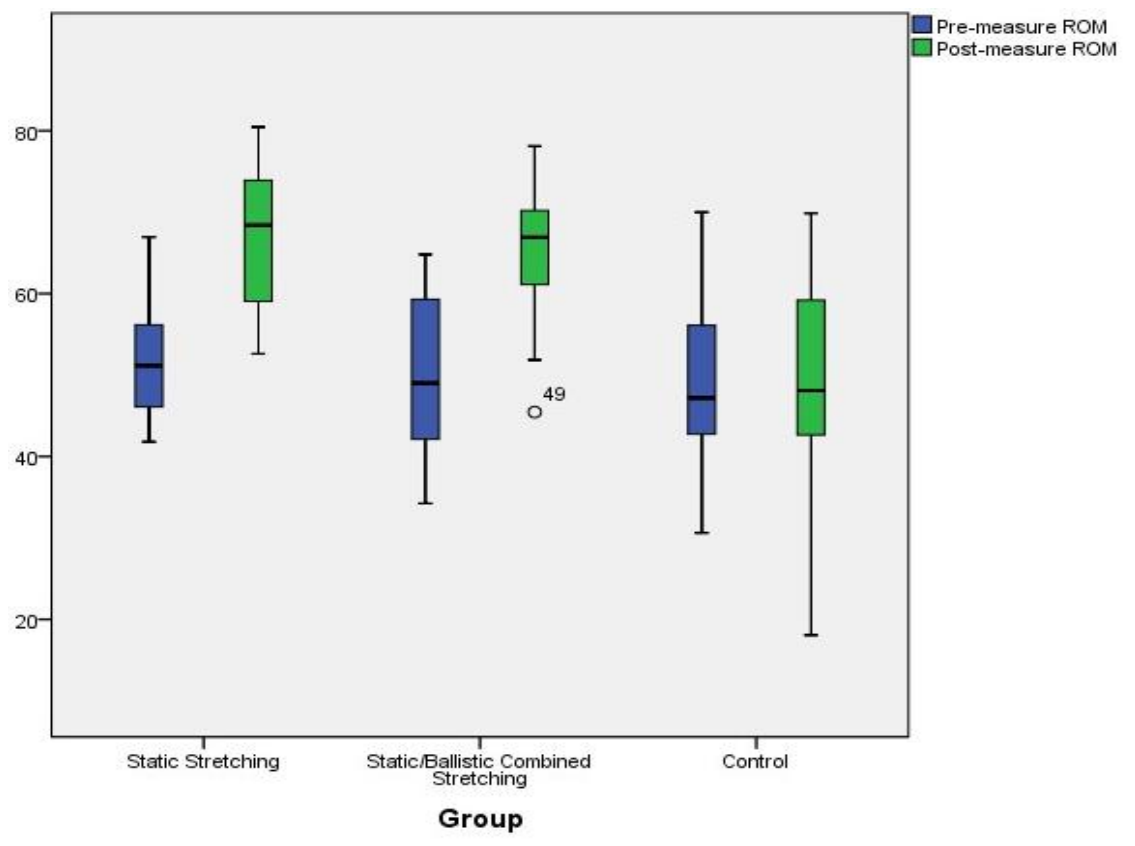

Brief non-ballistic active stretching through controlled active knee extension has been shown to improve range of motion, as demonstrated by Webright et al. ${ }^{17}$ By performing 30, 1-second stretches in a controlled manner, via active knee extension, twice daily for 6 weeks, subjects gained significantly, without any significant difference between active and static stretching at postmeasurement. ${ }^{17}$ In our CSBS group, subjects similarly performed 30-second stretching bouts, involving 5, 1-second active movements integrated into the 30-second bout, via gentle ballistic trunk flexion, twice daily for 2 weeks, and also gained significantly, without any significant difference between the static and combined static and ballistic stretching groups. Despite our method being coupled with 10 seconds of static stretching prior to and following the ballistic movements, our findings were consistent with the study by Webright et al study using 30 seconds and twice-daily stretching as the parameters for duration and frequency, respectively, and differed only in the length of program implementation, assuming that individually subjective stretching intensities were equal. ${ }^{17}$ 
Research focusing on the increase in passive ROM due to stretching has shown that significance is typically found by week 4 of a regular standardized program. 15,16 When determining any possible differences between a 2-week and a 4-week static stretching program, Davis et al found no significant gains at the 2-week mark, but did find significant results at week $4 .{ }^{16}$ It is likely that the significant differences we found in both intervention groups in only two weeks is related to the weekly volume of stretches. Davis et al evaluated stretches performed once daily, 3 days a week for 4 weeks, whereas this study evaluated stretches performed twice daily, every day for 2 weeks. ${ }^{16}$ Based on the research of Bandy et al, who found that significance cannot be found beyond one stretching bout per day, the only differing factor between Davis et al and our study would be the weekly and not the daily frequency.6.,17 However, Bandy et al studied multiple stretches performed in a day with 10 seconds of rest between, whereas our study investigated stretches performed with several hours in between, at opposite ends of the day. ${ }^{6}$ Regardless, in both parameters, the volume expressed was greater in this current study than for Davis et al. ${ }^{16}$ This demonstrates that with the right amount of volume, gains can become significant within 2 weeks. Future research should examine whether more flexibility can be achieved, and by how much, with the protocol in this study in a more traditionally studied 4-week period. We chose 2 stretches per day as this is what is typical for a collegiate athlete wanting to improve ROM.

\section{Limitations}

One of the limitations of this investigation was the lack of supervision of the subjects when performing each stretching session. A regular twice-daily laboratory setting was not feasible, but certain measures were taken to ensure quality of performance and adherence to the designed stretching schedule. All subjects received written and video instructions of the assigned stretching protocol to create standardization of presentation of proper technique and execution, which also allowed subjects the opportunity to frequently review these resources to maintain optimum quality. Additionally, all subjects agreed to receive two daily text messages, one in the morning and the other in the evening, to act as a reminder to avoid missed sessions. Subjects also were not directed to perform each morning and evening stretch at specified times each day, but rather were asked to make an effort to stretch as close to a regular time each day as possible, which caused differing lengths of time between stretching bouts from subject to subject. This is also due to feasibility on the part of the subjects, but also represents a more practical application of stretching by the general population in order to improve range of motion for fitness and performance purposes. Despite this limitation, all post-test measurement appointments were made for the same time as the pre-test measurement appointment to control for variance in muscle extensibility that can occur at different times of day.

Another limitation was the sample size. We utilized 43 total subjects, 15 control subjects and 28 subjects in the intervention groups, providing 83 total measurements, randomly divided into the 3 groups. Despite the limited number of subjects within each group, significant gains and effect sizes were achieved in both intervention groups when compared to the non-stretching control group. Furthermore, all subjects used were young, healthy adults with limited hamstring flexibility, from the general population.

\section{Assumptions}

It was assumed that each participant gave a complete effort to perform each stretching session and active knee ROM test to the best of their ability, and that the signed stretching logs accurately displayed subject compliance throughout the 2-week treatment period. It was also assumed that each subject adequately and equally understood the written and video instructions, allowing for equal quality stretching bouts for each subject throughout the treatment period, as well as from subject to subject.

\section{Future Research}

Future research should analyze the CSBS protocol used in this study in comparison to a second CSBS group, performing the same method but with 2 stretching bouts per day with 10 seconds of rest between the 2 bouts, as opposed to several hours of separation as performed in this study. This will demonstrate any differences that might take place when stretching bouts are performed close together or far apart, and will add to the work of Bandy et al who determined that no significance takes place when more than one stretch a day is performed. ${ }^{6}$ This may help clarify whether the findings of Bandy et al remain constant when the stretches are spread out over a day, rather than performed consecutively in a short period of time. Perhaps the gains made and the statistical significance achieved in both the SS and CSBS groups would be decreased when the two daily stretching sessions are scheduled close together, suggesting that multiple stretches performed in a single day may in fact be beneficial when spaced further apart, as has been done in this investigation.

Additionally, further research should also study any differences between a 2-week, twice-daily CSBS protocol and a 4-week, twice-daily CSBS protocol, also evaluating for differences in active knee ROM gains by week 2 as compared to week 4 to determine if one half of the 4-week program may be responsible for the majority of the total gains made.

Future research should also study the effects of the SS and CSBS stretching protocols on competitive athletes of varying ages and sports and the lasting effects of the stretching protocols.

(C) The Internet Journal of Allied Health Sciences and Practice, 2015 


\section{REFERENCES}

1. Weerapong P, Hume PA, Kolt GS. Stretching: Mechanisms and benefits for sport performance and injury prevention. Physical Therapy Reviews. 2004;9(4):189-206.

2. Ogura $\mathrm{Y}$, Miyahara $\mathrm{Y}$, Naito H, Katamoto $\mathrm{S}$, Aoki J. Duration of static stretching influences muscle force production in hamstring muscles. J Strength Cond Res. 2007 Aug;21(3):788-92. [PMID 17685679]

3. Wong del P, Chaouachi A, Lau PW, Behm DG. Short durations of static stretching when combined with dynamic stretching do not impair repeated sprints and agility. J Sports Sci Med. 2011 Jun 1;10(2):408-16. [PMID 24149890]

4. Little T, Williams AG. Effects of differential stretching protocols during warm-ups on high speed motor capacities in professional soccer players. J Strength Cond Res. 2006 Feb;20(1):203-7. [PMID 16503682]

5. Pope RP, Herbert RD, Kirwan JD, Graham BJ. A randomized trial of preexercise stretching for prevention of lower-limb injury. Med Sci Sports Exerc. 2000;32(2):271-7. [PMID 10694106]

6. Bandy WD, Irion JM, Briggler M. The effect of time and frequency of static stretching on flexibility of the hamstring muscles. Phys Ther. 1997;77(10):1090-6. [PMID 9327823]

7. deWeijer VC, Gorniak GC, Shamus E. The effect of static stretch and warm-up exercise on hamstring length over the course of 24 hours. J Orthop Sports Phys Ther. 2003 Dec;33(12): 727-33. [PMID 14743986]

8. Harvey L, Herbert R, Crosbie J. Does stretching induce lasting increases in joint ROM? A systematic review. Physiother Res Int. 2007;7(1): 1-13. [PMID 11992980]

9. Bandy WD, Irion JM, Briggler M. The effect of static stretch and dynamic range of motion training on the flexibility of the hamstring muscles. J Orthop Sports Phys Ther. 1998 Apr;27(4):295-300. [PMID 9549713]

10. Kumar CKK, Chakrabarty S. A comparative study of static stretching versus ballistic stretching on the flexibility of the hamstring muscles of athletes. British Journal of Sports Medicine. Sep 2010 Supp;44:i16.

11. Gregory JE, Brockett CL, Morgan DL, Whitehead NP, Proske U. Effect of eccentric muscle contractions on Golgi tendon organ responses to passive and active tension in the cat. J Physiol. 2002 Jan 1;538(Pt 1):209-18. [PMID 11773329]

12. Stephens JA, Reinking RM, Stuart DG. Tendon organs of cat medial gastrocnemius: responses to active and passive forces as a function of muscle length. J Physiol. 1975 Sep;38(5):1217-31. [PMID 1177014]

13. Swett JE, Schoultz TW. Mechanical transduction in the Golgi tendon organ: a hypothesis. Arch Ital Biol. 1975;113(4):37482. [PMID 1230097]

14. Avela J, Finni T, Liikavainio T, Niemelä E, Komi PV. Neural and mechanical responses of the triceps surae muscle group after $1 \mathrm{~h}$ of repeated fast passive stretches. J Appl. Physiol. 2004 Jun;96(6):2325-32. [PMID 14966020]

15. Chan SP, Hong Y, Robinson PD. Flexibility and passive resistance of the hamstrings of young adults using two different static stretching protocols. Scand J Med Sci Sports. 2001 Apr;11(2):81-6. [PMID 11252465]

16. Davis DS, Ashby PE, McCale KL, McQuain JA, Wine JM. The effectiveness of 3 stretching techniques on hamstring flexibility using consistent stretching parameters. J Strength Cond Res. 2005 Feb;19(1):27-32. [PMID 15705041]

17. Webright WG, Randolph BJ, Perrin DH. Comparison of nonballistic active knee extension in neural slump position and static stretch techniques on hamstring flexibility. J Orthop Sports Phys Ther. 1997 Jul;26(1):7-13. [PMID 9201636] 